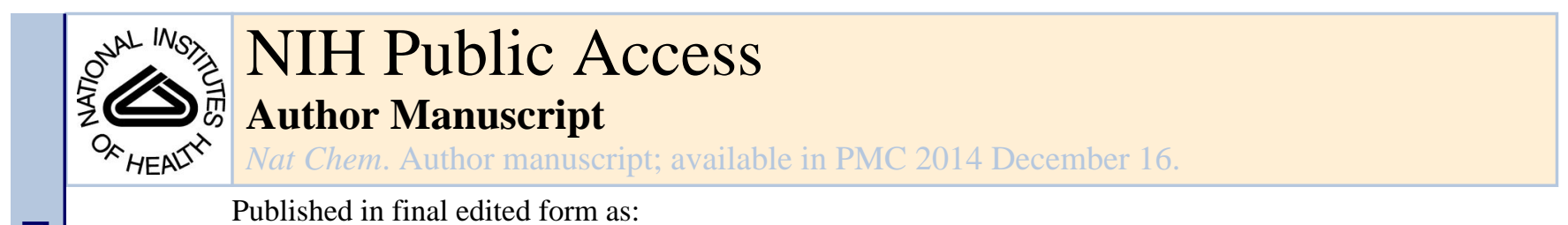

Published in final edited form as:

Nat Chem. ; 4(1): 13-14. doi:10.1038/nchem.1230.

\title{
Relieving PEGylation
}

\author{
Matthew C. Parrott and Joseph M. DeSimone \\ Department of Chemistry at the University of North Carolina at Chapel Hill, CB\# 3290, 257 \\ Caudill, Chapel Hill, North Carolina 27599-3290, USA.
}

\section{Abstract}

A new type of protein-polymer conjugate provides improved stability without detrimentally affecting bioactivity, and thus offers great potential for the development of new peptide-based drugs.

Currently, there are over 130 protein- or peptide-based therapeutics approved for clinical use $^{1}$. Insulin is probably the most well-known protein therapeutic - it is currently used by 171 million people worldwide for glucose regulation ${ }^{2}$. Protein therapeutics cause little or no immune response. Furthermore, they exhibit high disease specificity, and are usually effective at producing a positive outcome for most patients. With such a positive combination of desirable properties the recent increase in the popularity of protein- and peptide-based therapeutics is easily understood. These advantages can be attributed to a structure built from commonly occurring biological molecules that are well tolerated by the body, and its ability to perform specific functions without interfering with normal biological processes. Consequently, protein therapeutics has tremendous potential to manage and cure a number of complicated diseases.

Hindering the rapid progression of this field are the inherent sensitivities associated with proteins, including: thermal instability, rapid excretion from the body, degradation by proteolysis, and low solubility. A common method used to evade these limitations is the covalent attachment of poly(ethylene glycol) (PEG) to the protein - a technique known as PEGylation. PEG is widely known as an amphiphilic polymer (that is, having both hydrophilic and hydrophobic parts) that can improve water solubility, and increase native protein stability while simultaneously decreasing non-specific protein adsorption. This extends a protein's circulation time in the body, and the added steric bulk minimizes protein-protein interactions, which reduces the possibility of degradation ${ }^{3}$. The practice of PEGylation is generally regarded as the standard approach used to enhance protein properties, and has led to a number of therapeutics for the treatment of leukaemia, immunodeficiency diseases and hepatitis $\mathrm{C}$.

Unfortunately, the attachment of PEG to a protein is not without its own limitations. One of the most significant is the loss of biological activity. In the case of PEG-interferon a2a (Pegasys) - used in the treatment of hepatitis $\mathrm{C}$ - only $7 \%$ of the interferon a2a activity 
remains following the PEG modification ${ }^{4}$. Similar reductions in biological activity can be seen in PEGylated monoclonal antibodies and enzymes. The cause of this is postulated to be interference in receptor binding due to PEGylation ${ }^{5}$, either through simple steric crowding effects or through hydrophobic- hydrophobic interactions between the amphiphilic PEG and hydrophobic domains within the protein.

Writing in Nature Chemistry, Andrew Keefe and Shaoyi Jiang have identified a new polymeric material ${ }^{6}$ that outperforms PEGylation without sacrificing the bioactivity of the native protein. They developed a polymer that is more resistant to the non-specific adsorption of proteins ${ }^{7}$, which is composed of zwitterionic repeat units (each unit contains an equal number of anions and cations so that the polymer has an overall neutral charge). Analogous to PEG, a rationally designed zwitterionic polymer, when covalently attached to a protein, provides stability, prolonged circulation, reduced degradation and increased water solubility. The key difference between PEG and a poly(zwitterion) is the surface properties associated with each polymer. PEG interacts with water through hydrogen bonding leading to a highly viscous, amphiphilic coating, whereas poly(zwitterion)s associate with water through strong ionic interactions, creating a super-hydrophilic surface that promotes protein-substrate affinity and in certain cases enhances the biological response (Fig. 1).

Keefe and Jiang investigated multiple variables to compare the biological activity of the proteolytic digestive enzyme a-chymotrypsin affer PEGylation or following the attachment of a poly(zwitterion) based on betaine, known as poly(carboxybetaine). This was carried out in the presence of a high concentration of urea, which is known to denature proteins. The addition of a single PEG chain showed negligible change in bioactivity when compared with the native enzyme. Conversely, the addition of one poly(zwitterionic) polymer showed a significant increase in bioactivity, indicating enhanced protein stability under unfavourable conditions. Furthermore, the thermal stability of the protein-polymer conjugates were investigated at elevated temperatures. The poly(zwitterionic) conjugates demonstrated consistent bioactivity at temperatures $\sim 5^{\circ} \mathrm{C}$ higher than both the native protein and the PEGylated protein. More remarkably, the binding affinity between a small peptide substrate and the protein-polymer conjugates was measured as a function of the number of polymer chains attached to the protein. As the number of PEG chains increased the binding affinity decreased. In contrast, as the number of poly(zwitterion) polymers increased, the binding affinities remained similar to the native protein, or in the case of high-molecular-weight poly(zwitterion) polymers, the binding affinity actually improved. This verified that PEGylation not only imparts steric hindrance, but also inhibits the protein-substrate interaction. Meanwhile, a rationally designed poly(zwitterion) promotes the proteinsubstrate interaction.

The application of poly(zwitterion)-functionalized proteins provides an alternative to PEGylation. For the first time, a protein can be functionalized with a polymer for improved water solubility, increased thermal stability, and reduced degradation without sacrificing bioactivity. Not only that, this research also provides a path to more robust protein-polymer conjugates. This could have a significant impact on emerging protein therapeutics, and an even broader impact on managing complex diseases. 


\section{References}

1. Leader B, Baca QJ, Golan DE. Nature Rev. Drug Discov. 2008; 7:21-39. [PubMed: 18097458]

2. Wild S, Roglic G, Green A, Sicree R, King H. Diabetes Care. 2004; 27:1047-1053. [PubMed: 15111519]

3. Veronese FM, Pasut G. Drug Discov. Today. 2005; 10:1451-1458. [PubMed: 16243265]

4. Veronese FM, Mero A. Biodrugs. 2008; 22:315-329. [PubMed: 18778113]

5. Bailon P, et al. Bioconjugate Chem. 2001; 12:195-202.

6. Keefe AJ, Jiang S. Nature Chem. 2012; 4:59-63. [PubMed: 22169873]

7. Yang W, Zhang L, Wang S, White AD, Jiang S. Biomaterials. 2009; 30:5617-5621. [PubMed: 19595457] 

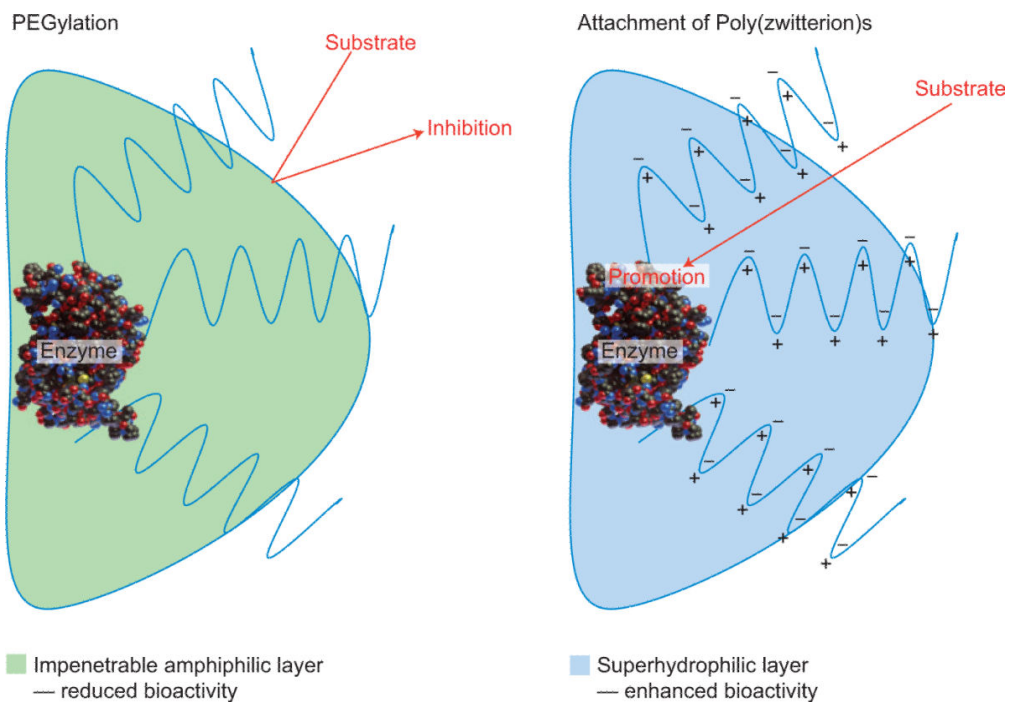

Figure 1.

The effect of polymer conjugation on peptide therapeutics. Attaching PEG chains imposes steric hindrance and introduces interactions that compete with substrate binding. Attaching a poly(zwitterion) can help to draw water away from the active site and shift the equilibrium to favour substrate binding. 